\title{
Velocity Dependence of Friction of Confined Hydrocarbons
}

\author{
Sivebæk, Ion Marius; Samoilov, Vladimir N.; Persson, Bo N. J.
}

Published in:

Langmuir

Link to article, DOI:

$10.1021 / \mathrm{la904476d}$

Publication date:

2010

Document Version

Publisher's PDF, also known as Version of record

Link back to DTU Orbit

Citation (APA):

Sivebæk, I. M., Samoilov, V. N., \& Persson, B. N. J. (2010). Velocity Dependence of Friction of Confined Hydrocarbons. Langmuir, 26(11), 8721-8728. https://doi.org/10.1021/la904476d

\section{General rights}

Copyright and moral rights for the publications made accessible in the public portal are retained by the authors and/or other copyright owners and it is a condition of accessing publications that users recognise and abide by the legal requirements associated with these rights.

- Users may download and print one copy of any publication from the public portal for the purpose of private study or research.

- You may not further distribute the material or use it for any profit-making activity or commercial gain

- You may freely distribute the URL identifying the publication in the public portal

If you believe that this document breaches copyright please contact us providing details, and we will remove access to the work immediately and investigate your claim 


\title{
Velocity Dependence of Friction of Confined Hydrocarbons
}

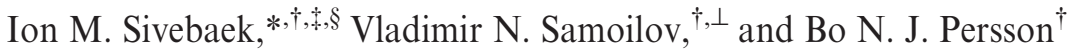 \\ ${ }^{\dagger} I F F$, FZ-Jülich, 52425 Jülich, Germany, ${ }^{\ddagger}$ Novo Nordisk A/S, Research and Development, DK-3400 Hillerod, \\ Denmark, ${ }^{\S}$ Mechanical Engineering Department, Technical University of Denmark, DK-2800 Lyngby, \\ Denmark, and ${ }^{\perp}$ Physics Faculty, Moscow State University, 117234 Moscow, Russia
}

Received November 26, 2009. Revised Manuscript Received February 17, 2010

\begin{abstract}
We present molecular dynamics friction calculations for confined hydrocarbon "polymer" solids with molecular lengths from 20 to 1400 carbon atoms. Two cases are considered: (a) polymer sliding against a hard substrate and (b) polymer sliding on polymer. We discuss the velocity dependence of the frictional shear stress for both cases. In our simulations, the polymer films are very thin $(\sim 3 \mathrm{~nm})$, and the solid walls are connected to a thermostat at a short distance from the polymer slab. Under these circumstances we find that frictional heating effects are not important, and the effective temperature in the polymer film is always close to the thermostat temperature. In the first setup (a), for hydrocarbons with molecular lengths from 60 to 1400 carbon atoms, the shear stresses are nearly independent of molecular length, but for the shortest hydrocarbon $\mathrm{C}_{20} \mathrm{H}_{42}$ the frictional shear stress is lower. In all cases the frictional shear stress increases monotonically with the sliding velocity. For polymer sliding on polymer (case b) the friction is much larger, and the velocity dependence is more complex. For hydrocarbons with molecular lengths from 60 to $140 \mathrm{C}$ atoms, the number of monolayers of lubricant increases (abruptly) with increasing sliding velocity (from 6 to 7 layers), leading to a decrease of the friction. Before and after the layering transition, the frictional shear stresses are nearly proportional to the logarithm of sliding velocity. For the longest hydrocarbon (1400 C atoms) the friction shows no dependence on the sliding velocity, and for the shortest hydrocarbon $(20 \mathrm{C}$ atoms $)$ the frictional shear stress increases nearly linearly with the sliding velocity.
\end{abstract}

\section{Introduction}

Friction between solids is a very important phenomenon in biology and technology, ${ }^{1}$ and it is very common in nature. Static friction always involves the coexistence of different metastable configurations at microscopic level. When one surface slides on the other at low speed, first there is a loading phase during which the actual configuration stores elastic energy. Then, when the stored energy is large enough, an instability arises: ${ }^{2-4}$ the system jumps abruptly to another configuration and releases elastic energy into irregular heat motion. The exact way of how the energy is dissipated usually does not influence the sliding friction force, provided that the dissipation is fast enough to happen before the next sliding event.

There are many possible origins of elastic instabilities; e.g., they may involve individual molecules or, more likely, groups of molecules or "patches" at the interface, which have been named stress domains. ${ }^{5-8}$ Since the local rearrangements usually occur at different times in an incoherent manner, at the macroscopic scale the sliding motion may appear smooth without stick-slip oscillations. However, this is always a result of self-averaging, and at the atomistic level stick-slip motion will almost always occur (except for incommensurate systems with weak interactions). Moreover,

*To whom correspondence should be addressed. E-mail: ions $a$ novonordisk.com.

(1) Persson, B. N. J. Sliding Friction: Physical Principles and Applications, 2nd ed.; Springer: Heidelberg, 2000.

(2) Aubry, J. J. Phys. (Paris) 1983, 44, 147.

(3) Persson, B. N. J.; Tosatti, E. Solid State Commun. 1999, 109, 739.

(4) Caroli, C.; Nozieres, P. In Physics of Sliding Friction; Persson, B. N. J., Tosatti, E., Eds.; Kluwer: Dordrecht, 1996.

(5) Persson, B. N. J.; Albohr, O.; Mancosu, F.; Peveri, V.; Samoilov, V. N.; Sivebaek, I. M. Wear 2003, 254, 835.

(6) Persson, B. N. J. Phys. Rev. 1995, B51, 13568

(7) Caroli, C.; Nozieres, P. Eur. Phys. J. 1998, B4, 233

(8) Baumberger, T.; Caroli, C. Adv. Phvs. 2006, 55, 279. at least at zero temperature, the friction force does not vanish in the limit of sliding speed $v \rightarrow 0$, but it tends to some finite value which depends on the average energy stored during the loading events and the atomic slip distance.

A logarithmic velocity dependence of the frictional shear stress was observed in many experiments but usually for sliding at low velocities (up to $\approx 20 \mu \mathrm{m} / \mathrm{s}$ ). ${ }^{9}$ Still, in some experiments the logarithmic velocity dependence of the frictional shear stress was observed also for higher velocities (up to $1 \mathrm{~mm} / \mathrm{s}$ ). ${ }^{10}$ Comparing experimental results with the existing theoretical models $\mathrm{s}^{5,9,11}$ shows that the logarithmic dependence could be well described in the models accounting for thermal activation effects. Thus, thermal fluctuations may induce jump of atoms (or rather group of atoms) at the sliding interface from one equilibrium position to the next one along the reaction path. The resulting stress-aided thermally activated effect leads to a logarithmic increase of friction with the velocity at low velocities. Thermal activation is more efficient at low velocities, where the system spends long time in each potential well, and consequently, the probability to thermally activate the processes of atoms hopping is higher.

When a polymer (or a long-chain alkane) is sheared between two surfaces, the shear stress often does not exhibit a linear dependence of the logarithm of the sliding velocity. This has been established both experimentally ${ }^{12-14}$ and in simulations. ${ }^{15-17}$ This observation may be due to the interdiffusion of chain segments between

(9) Riedo, E.; Gnecco, E. Nanotechnologv 2004, 15, S288.

(10) Tocha, E.; Stefanski, T.; Schoenherr, H.; Vancso, G. J. Rev. Sci. Instrum. 2005, 76, 083704 .

(11) Sang, Y.; Dube, M.; Grant, M. Phys. Rev. 2008, E77, 036123.

(12) Drummond, C.; Alcantar, N.; Israelachvili, J. Phys. Rev. 2002, E66, 011705.

(13) Bureau, L.; Baumberger, T.; Caroli, C. Eur. Phys. J. 2006, E19, 163.

(14) Qian, L.-M.; Luengo, G.; Perez, E. Europhvs. Lett. 2003, 61, 268.

(15) Subbotin, A.; Semenov, A.; Doi, M. Phys. Rev. 1997, E56, 623.

(16) Thompson, P. A.; Robbins, M. O.; Grest, G. S. Isr. J. Chem. 1995, 35, 93.

(17) He, G.; Robbins, M. O. Tribol. Lett. 2001, 10, 7. 
the polymer layers by the long molecules. Thus, the formation of "bridges" is presumably affected by a change in the sliding velocity, and in certain regimes it is possible to have decreasing shear stress with increasing sliding velocity due to the disappearing of bridges across the sliding interface. ${ }^{14,15}$ This picture was first suggested in the context of rubber friction by Schallamach. ${ }^{18}$

In this paper we present molecular dynamics friction calculations for confined hydrocarbon solids with molecular lengths from 20 to $1400 \mathrm{C}$ atoms. Two cases are considered: (a) polymer sliding against a hard substrate and (b) polymer sliding on polymer. We discuss the velocity dependence of the frictional shear stress for both cases. We compare results obtained at room temperature and very low temperature (approaching $0 \mathrm{~K}$ ). In the latter calculations no thermal activation can occur.

In our simulations, the polymer films are very thin $(\sim 3 \mathrm{~nm})$, and the solid walls are connected to a thermostat at a short distance from the polymer slab. Under these circumstances we find that frictional heating effects are not important, and the effective temperature in the polymer film is always close to the thermostat temperature. In most practical situations the temperature is not fixed at planes close to the interface, and during sliding at high enough velocities for a long enough time, the local temperature at the sliding interface may be so high as to locally melt the polymer surfaces. The physical processes occurring at the sliding interface in these cases may be a combination of the effects studied in this paper and the influence of the increased temperature.

\section{The Model}

In this paper we present computer simulation results about the frictional behavior of linear hydrocarbons under applied pressure. Our model is similar to those described in earlier work, ${ }^{19-22}$ but we review its main features here. We consider a block and a substrate with atomically flat surfaces separated by a polymer slab. Two cases are considered: (a) polymer sliding against a hard substrate which we will denote as "metal" for simplicity (the metal-polymer case) and (b) polymer sliding on polymer (the polymer-polymer case).

The solid walls are treated as single layers of "atoms" bound to rigid flat surfaces by springs corresponding to the long-range elastic properties of $50 \AA$ thick solid slabs. This was performed similar to our earlier papers. ${ }^{20}$ The atoms in the bottom layer of the block form a simple square lattice with lattice constant $a$. In the following, periodic boundary conditions are assumed in the $x y$ plane. The atoms of the block interact with each other via "stiff" springs and execute bending and stretching motion characterized by a bending force constant $k_{\mathrm{OB}}$ and a stretching force constant $k_{0}$, respectively. Moreover, each atom is connected to the upper rigid surface profile by "soft" elastic springs, of bending force constant $k_{1 \mathrm{~B}}$ and stretching force constant $k_{1}$. The numerical values of all these force constants $k_{0}, k_{0 \mathrm{~B}}, k_{1}$, and $k_{1 \mathrm{~B}}$ are determined in such a way to mimic the elastic response of the entire slab. If we apply a shear stress $\sigma$ to the slab, the resulting strain $\varepsilon$ is given by $\sigma=2 G \varepsilon$, where $G=E / 2(1+v)$ is the shear modulus, $E$ is the elastic modulus, and $v$ the Poisson ratio. If we write the shear strain as $\varepsilon=\Delta / 2 a$, then $\sigma=k_{0 \mathrm{~B}} \Delta / a^{2}=G \Delta / a$, and we get $k_{0 \mathrm{~B}}=G a$.

(18) Schallamach, A. Wear 1963, 6, 375

(19) Persson, B. N. J.; Ballone, P. J. Chem. Phvs. 2000, 112, 9524.

(20) Persson, B. N. J.; Samoilov, V. N.; Zilberman, S.; Nitzan, A. J. Chem. Phvs. 2002, 117, 3897

(21) Sivebaek, I. M.; Samoilov, V. N.; Persson, B. N. J. J. Chem. Phvs. 2003, 119, 2314.

(22) Sivebaek, I. M.; Samoilov, V. N.; Persson, B. N. J. Eur. Phvs. J. E 2008, 27,
Similarly, we obtain that $k_{0}=E a$. Next, let us consider an elastic slab of thickness $W$. If we apply a shear stress $\sigma$, we get the relative displacement $x$ so that the strain is $\varepsilon=x / 2 W$. Thus, $\sigma=G x / W$, which must equal $k_{1 \mathrm{~B}} x / a^{2}$ and hence $k_{1 \mathrm{~B}}=G a^{2} / W$. In a similar way one can obtain $k_{1}=E a^{2} / W$.

The substrate is treated in a similar way as the block. The space between the block and the substrate is occupied by a slab (6-8 monolayers) of the hydrocarbons.

For the case of sliding of polymer on "metal", all molecules are adsorbed on the block surface only due to different parameters of interaction of alkane molecules with the walls, whereas for the case of sliding of polymer on polymer about half of the molecules adsorbed on the block surface and half on the substrate surface. The block with adsorbed polymer slab was put into contact with the substrate surface in the first case, and two solids with adsorbed polymer slabs were put into contact in the second case. When the temperature was equal to the thermostat temperature (usually $300 \mathrm{~K}$ ) everywhere, we started to move the upper block surface. We also conducted calculations for the temperature of solid walls equal to $0 \mathrm{~K}$ in order to compare the sliding friction behavior at $300 \mathrm{~K}$ with that in the absence of thermal activation at $T=0 \mathrm{~K}$. The temperature was also varied from 300 to $550 \mathrm{~K}$ to study the effect of melting on the shear stress.

Linear alkanes $\mathrm{C}_{n} \mathrm{H}_{2 n+2}$ (with $n$ ranging from 20 to 1400) were used as "lubricant" in the present calculations. The $\mathrm{CH}_{2} / \mathrm{CH}_{3}$ beads are treated in the united atom representation. ${ }^{23,24}$ The Lennard-Jones potential was used to model the interaction between beads of different chains

$$
U(r)=4 \varepsilon_{0}\left[\left(\frac{r_{0}}{r}\right)^{12}-\alpha\left(\frac{r_{0}}{r}\right)^{6}\right]
$$

and the same potential with modified parameters $\left(\varepsilon_{1}, r_{1}\right)$ was used for the interaction of each bead with the substrate and block atoms. (Note that using $\alpha$ in $U(r)$ is equivalent to change the values of $\varepsilon_{0}$ and $r_{0}$ to $\varepsilon_{0}^{\prime}$ and $r_{0}^{\prime}$ with $\varepsilon_{0}^{\prime}=\varepsilon_{0} \alpha^{2}$ and $r_{0}^{\prime}=r_{0} \alpha^{-1 / 6}$, respectively.) The parameters were $\varepsilon_{0}=5.12 \mathrm{meV}$ for both the interior and the end beads, and $r_{0}=3.905 \AA$ and $\alpha=1$. For the interactions within the $\mathrm{C}_{n} \mathrm{H}_{2 n+2}$ molecules we used the standard OPLS model, ${ }^{23,24}$ including flexible bonds, bond bending, and torsion interaction, which results in bulk properties in good agreement with experimental data. Atomic mass 14 (for interior $\mathrm{CH}_{2}$ beads) and 15 (for the $\mathrm{CH}_{3}$ end groups) were used. Within a $\mathrm{C}_{n} \mathrm{H}_{2 n+2}$ chain we assume that nearest-neighbor $\mathrm{C}$ atoms are connected via springs with the spring constant $k$, which was chosen equal to $10 \mathrm{~N} / \mathrm{m}$. Note that this value is 1 order of magnitude smaller than the optimized $450 \mathrm{~N} / \mathrm{m}^{23}$ and was chosen such to facilitate a reasonable time step of $1 \mathrm{fs}$. We used an angle bending interaction of the form $E(\cos \theta) / k_{\mathrm{B}}=1 / 2 k_{\text {bend }}(\cos \theta-$ $\left.\cos \theta_{0}\right)^{2}$ with $k_{\text {bend }}=62543 \mathrm{~K}$ and $\theta_{0}=2.0001 \mathrm{rad}$. For the dihedral interaction we used the functional form in term of a cosine Fourier series $E(\phi) / k_{\mathrm{B}}=\sum_{i=0}^{3} c_{i} \cos ^{i}(\phi)$ with parameters $c_{0}=1009.99 \mathrm{~K}, c_{1}=2018.95 \mathrm{~K}, c_{2}=136.37 \mathrm{~K}$, and $c_{3}=$ $-3165.30 \mathrm{~K}$. Internal beads of separation greater than three units are treated similarly as beads from different chains.

For polymer sliding on polymer we need the polymer-metal bond to be so strong that no slip occurs at these interfaces. This is the case with $r_{1}=3.28 \AA, \varepsilon_{1}=40 \mathrm{meV}$, and $\alpha=3$. We also did some simulations with $\alpha=2$, but in this case some slip was observed at the polymer-metal interface. For sliding of polymer

(23) Jorgensen, W. L.; Madura, J. D.; Swenson, C. J. J. Am. Chem. Soc. 1984, 106,6638 .

(24) Dysthe, D. K.; Fuchs, A. H.; Rousseau, B. J. Chem. Phvs. 2000, 112, 7581. 


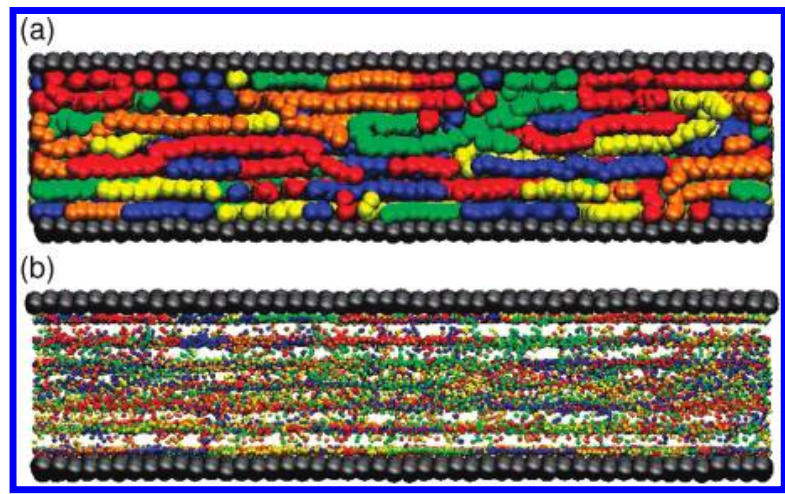

Figure 1. Snapshot picture of the $\mathrm{C}_{100} \mathrm{H}_{202}$ polymer slab at the sliding velocity $v=10 \mathrm{~m} / \mathrm{s}$ and background temperature $T=$ $300 \mathrm{~K}$. (a) The molecules are (arbitrarily) colored in order to better observe the shear alignment of the chains. (b) The same as in (a) but with atoms presented as points in order to observe layering in the system. Seven monolayers of molecules are clearly seen.

on "metal" we used the same parameters as above for the polymer-block interaction but with $\alpha=1$, and for the polymersubstrate interaction we used $\alpha=1$ and $\varepsilon_{1}=10 \mathrm{meV}$.

The choice of higher values of $\varepsilon_{1}$ compared to $\varepsilon_{0}$ reflects the stronger (van der Waals) interaction between the beads and the "metal" surfaces than between the bead units of different lubricant molecules (this stronger interaction results from the higher electron density in the metals). The lattice spacings of the block and of the substrate are $a=b=2.6 \AA$.

Note that for polymer sliding on polymer the block and the substrate were identical in structure and interactions with the hydrocarbon molecules. This was not the case for polymer sliding on "metal". Here asymmetry was introduced by using different $\varepsilon_{1}$ for the polymer-block interaction $\left(\varepsilon_{1}=40 \mathrm{meV}\right)$ and the polymer-substrate interaction $\left(\varepsilon_{1}=10 \mathrm{meV}\right)$, so that the slipping events were to be observed mainly at this interface.

We used linear alkane molecules with the number of carbon atoms 20, 60, 100, 140, and 1400 as lubricant. The number of molecules was equal to $14,143,200,333$, and 1000 for the $\mathrm{C}_{1400} \mathrm{H}_{2802}, \mathrm{C}_{140} \mathrm{H}_{282}, \mathrm{C}_{100} \mathrm{H}_{202}, \mathrm{C}_{60} \mathrm{H}_{122}$, and $\mathrm{C}_{20} \mathrm{H}_{42}$ systems, respectively. This gave from 6 to 8 monolayers of lubricant molecules between the solid surfaces. The (nominal) squeezing pressure $p_{0}$ was usually $10 \mathrm{MPa}$.

As an illustration, in Figure 1 we show the contact between a flat elastic block (top) and a flat elastic substrate (bottom). The polymer slab ( $\sim 30 \AA$ thick $)$ is in between them. Only the interfacial block and substrate atoms and polymer atoms are shown.

The results in the following are given for systems that are run in at the given sliding velocities. Running in of long chain molecules is difficult due to chain entanglements. We consider a system to be run in when the averaged shear stress remains constant just as it is shown in Figure 4 of ref 22 after 16 ns of sliding.

Finally, we note that for the rather thick and somewhat disordered hydrocarbon films we use the fact that polymers cannot be squeezed out from the interface is not so important. In many earlier studies we have used curved surfaces in order to study squeeze-out of thin fluid films. ${ }^{21}$ Another way to take into account squeeze-out has been used in work by Gao and Landman. ${ }^{25}$

\section{Polymer on "Metal"}

In Figure 2 we show the dependence of the shear stress on the sliding velocity for the polymer slabs sliding on "metal" at the

(25) Gao, J. P.; Luedtke, W. D.; Landman, U. Phvs. Rev. Lett. 1997, 79, 705.

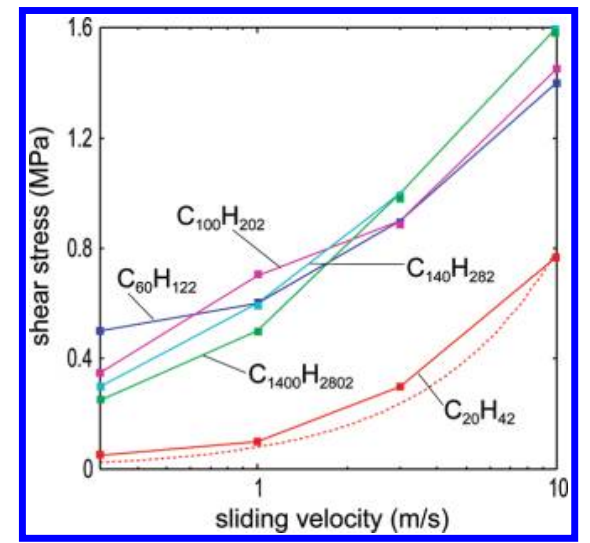

Figure 2. Dependence of the shear stress on the sliding velocity for the polymers from $\mathrm{C}_{20} \mathrm{H}_{42}$ to $\mathrm{C}_{1400} \mathrm{H}_{2802}$ sliding on "metal" at the normal pressure $10 \mathrm{MPa}$ and temperature $300 \mathrm{~K}$. The dotted line is a linear fit to the $\mathrm{C}_{20} \mathrm{H}_{42}$ curve.

applied pressure $p=10 \mathrm{MPa}$. For $\mathrm{C}_{20} \mathrm{H}_{42}$ the lubricant behaves liquidlike with the shear stress nearly proportional to the sliding velocity (see dashed curve in Figure 2). In particular, as the sliding velocity approaches zero, the shear stress for $\mathrm{C}_{20} \mathrm{H}_{42}$ becomes very small, reflecting the fact that this polymer is close to the liquid state: the $\mathrm{C}_{20} \mathrm{H}_{42}$ (eicosane) system is at $300 \mathrm{~K}$ (the temperature of the thermostat), which is very close to the melting point $(\approx 310 \mathrm{~K})$ of this polymer. We also performed simulations at the (thermostat) temperatures 200 and $0 \mathrm{~K}$, and in these cases the friction is considerably higher ( $\sim 9.6$ times higher at the temperature $0 \mathrm{~K}$ for the sliding velocity $v=0.3 \mathrm{~m} / \mathrm{s}$ ).

For longer chain hydrocarbon molecules we found that the polymer films behave more solidlike at $300 \mathrm{~K}$, with nonvanishing kinetic friction as the sliding velocity approaches zero. This is due to the higher melting point of the longer chain polymer systems. For the $\mathrm{C}_{100} \mathrm{H}_{202}$ system the frictional shear stress $\sigma_{\mathrm{f}}=0.33 \mathrm{MPa}$ for $T=300 \mathrm{~K}$ and $\sigma_{\mathrm{f}}=0.72 \mathrm{MPa}$ for $T=0 \mathrm{~K}$ for the sliding velocity $v=0.3 \mathrm{~m} / \mathrm{s}$. This increase in $\sigma_{\mathrm{f}}$ can be attributed to the absence of thermal activation at $T=0 \mathrm{~K}$. In general, at low sliding velocities the shear stress increases greatly much more than for the higher sliding velocities when the temperature is decreased from 300 to $0 \mathrm{~K}$. This is due to the fact that the probability to activate the processes of atoms hopping is higher at low velocities due to the longer time the system spends in each local potential well along the reaction coordinate.

Atomistic stick-slip events occur at the sliding interface between the polymer and the substrate. The period of oscillations of the shear stress as a function of $x$-coordinate of the block is exactly $2.6 \AA$, which is one lattice unit (of the substrate) in the sliding direction. Temperature-dependent stick-slip has also been observed by Baljon and Robbins. ${ }^{26}$

We emphasize the importance of the temperature (or thermal fluctuations) on the process of "going over the barrier". Thus, at zero temperature, the external applied tangential force (or stress) alone pulls the system over the lateral pinning barriers, and this happens everywhere simultaneously. At high sliding velocities thermal effect should be rather unimportant. However, for small sliding velocities, thermal fluctuations will be very important. In this case slip will not occur everywhere simultaneously, but small nanometer-sized interfacial regions of linear size $D$ will be individually pinned and perform stress-aided thermally induced jump from one pinned state to another (local interfacial rearrangement processes). (Note that thermal effects can only become

(26) Baljon, A. R. C.; Robbins, M. O. Science 1996, 271, 482. 


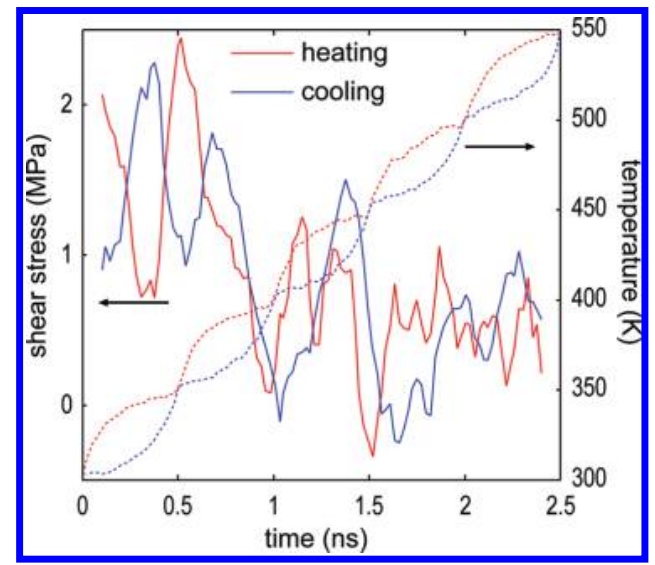

Figure 3. Shear stress for $\mathrm{C}_{140} \mathrm{H}_{282}$ polymer sliding on metal at the sliding velocity $v=10 \mathrm{~m} / \mathrm{s}$ and the normal pressure $p=10 \mathrm{MPa}$ when ramping the temperature $T$ in steps of $50 \mathrm{~K}$ from 300 to $550 \mathrm{~K}$ (heating) and from 550 to $300 \mathrm{~K}$ (cooling).

important for small (nanometer-sized $D$ ) regions, since simultaneous going-over-the-barrier everywhere requires infinitely large energy for an infinite system, except, perhaps for an incommensurate interface.) This process has been studied in detail both theoretically ${ }^{6,17,18,27,28}$ and experimentally. $8,29,30$

We now study the frictional behavior of the hydrocarbon films $\mathrm{C}_{100} \mathrm{H}_{202}$ and $\mathrm{C}_{140} \mathrm{H}_{282}$, when the temperature is increased above the melting temperatures. In Figure 3 we show the shear stress for the $\mathrm{C}_{140} \mathrm{H}_{282}$ polymer film (at the sliding velocity $v=10 \mathrm{~m} / \mathrm{s}$ and the normal pressure $p=10 \mathrm{MPa}$ ) when increasing the thermostat temperature $T$ by steps equal to $50 \mathrm{~K}$ from 300 to $550 \mathrm{~K}$ and then decreasing it back to $300 \mathrm{~K}$. Each $50 \mathrm{~K}$ temperature step was $0.5 \mathrm{~ns}$ long. During heating the shear stress decreases abruptly when the temperature is raised above the melting temperature $T_{\mathrm{m}}=383 \mathrm{~K}$ for $\mathrm{C}_{140} \mathrm{H}_{282}$ polymer. The frictional shear stress for the film in the liquidlike state is $\sim 3$ times lower than for the solid film just below melting.

For temperatures well above the melting point the molecules in the center of the polymer film are disordered as expected for the liquid state of the lubricant. In Figure 4 we show the density distribution and the average velocity $v_{x}$ of the $\mathrm{C}$ atoms along the distance between the substrate and the block ( $z$-direction) for the $\mathrm{C}_{100} \mathrm{H}_{202}$ polymer film. The results are for $T=300$ and $450 \mathrm{~K}$. At $T=300 \mathrm{~K}$ the film is seven monolayers thick, but at $T=450 \mathrm{~K}$, due to the thermal expansion, the system has eight monolayers. In the center the molecules are disordered and the layers of molecules blurred.

For $300 \mathrm{~K}$ the average velocity changes abruptly at the substrate-lubricant interface (see Figure 4a), so the slip occurs between the substrate and the first monolayer of molecules of the polymer film. Thus, the whole polymer film is bound to the block and moves with the average velocity $v_{x} \approx 10 \mathrm{~m} / \mathrm{s}$, i.e., with velocity of the block. For $T=450 \mathrm{~K}$ most of the slip also occurs at the polymer-substrate interface (see Figure 4 b), but a small slip (slip velocity $v \approx 1 \mathrm{~m} / \mathrm{s}$ ) also occurs at the polymer-block interface. Thus, all monolayers of the polymer film move with the average velocity $v_{x} \approx 9 \mathrm{~m} / \mathrm{s}$. The amount of slip is different at the two walls

(27) Briscoe, B. J. In Fundamentals of Friction: Macroscopic and Microscopic Processes; Singer, I. L., Pollock, H. M., Eds.; NATO ASI Series E: Applied Sciences Vol. 220; Kluwer Academic Publishers: Dordrecht, 1992.

(28) Persson, B. N. J.; Volokitin, A. I. Eur. Phys. J. 2006, E21, 69.

(29) (a) Drummond, C.; Israelachvili, J.; Richetti, P. Phys. Rev. 2003, E67, 066110. (b) Drummond, C.; Elezgaray, J.; Richetti, P. Europhvs. Lett 2002, $58,503$.

(30) (a) Baumberger, T.; Caroli, C.; Ronsin, O. Eur. Phys. J. 2003, E11, 85. (b) Ronsin, O.; Coeyrehourcq, K. L. Proc. R. Soc. London. Ser. A 2001, 457, 1277.

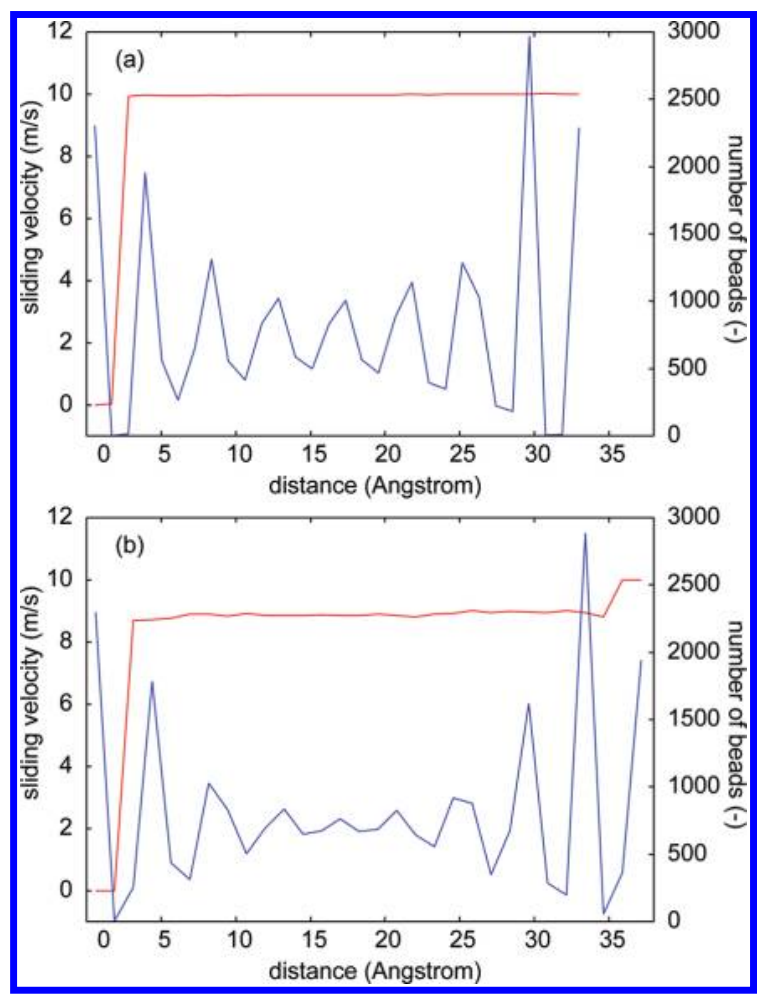

Figure 4. Distributions of the average number and the average velocity $v_{x}$ (in the sliding direction) of lubricant $\mathrm{C}$ atoms along the distance between the substrate and the block for $\mathrm{C}_{100} \mathrm{H}_{202}$ metal-polymer sliding at velocity of the block $v=10 \mathrm{~m} / \mathrm{s}$ for the normal pressure $p=10 \mathrm{MPa}$ for the temperature (a) $300 \mathrm{~K}$ and (b) $450 \mathrm{~K}$. In the latter case the slip also occurs between the block and the last monolayer of lubricant molecules due to thermal fluctuations. The very left and very right maximum of the both density distributions correspond to the substrate and the block atom layers.

due to the difference of $\varepsilon_{1}=40 \mathrm{meV}$ used for the polymer-block interaction and $\varepsilon_{1}=10 \mathrm{meV}$ used for the polymer-substrate interaction. The slip at the polymer-block interface is due to the applied shear stress and thermal fluctuations. At the lower temperature $300 \mathrm{~K}$ the thermal fluctuations are not strong enough to overcome the relatively large atomic corrugation at the polymer-block interface. Friction is a stress-aided thermally activated process, and in the present case when the temperature increases from $T=300$ to $450 \mathrm{~K}$, the shear stress drops by a factor of $\sim 2$.

The effective corrugation of the interaction potential experienced by the molecules at the sliding interface is the most important parameter influencing the magnitude of the friction and the dependence on the external (squeezing) pressure. Indeed, the fact that the lattice constant of the substrate is much smaller than the size of the polymer molecules (and the polymer persistence length), and also very different from the natural separation between the polymer molecules, implies that the effective corrugation of the interaction potential between the polymer and the "metal" substrate will be very small, and this explains the small friction observed in this case compared to the case when the slip occurs at the polymer-polymer interface. ${ }^{22,31}$

\section{Polymer on Polymer}

When a polymeric film is strongly attached to the block and substrate surfaces, sliding of the block will induce a shearing of the

(31) Thompson, P. A.; Robbins, M. O. Phys. Rev. 1990, A41, 6830. 


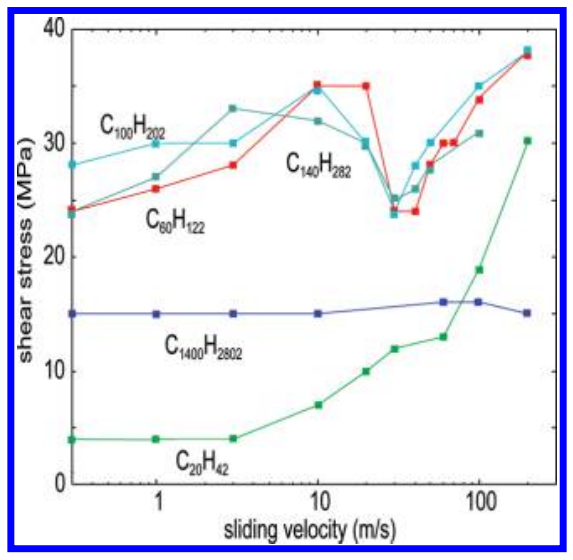

Figure 5. Shear stress as a function of the sliding velocity for all the investigated systems. The normal pressure is $10 \mathrm{MPa}$.

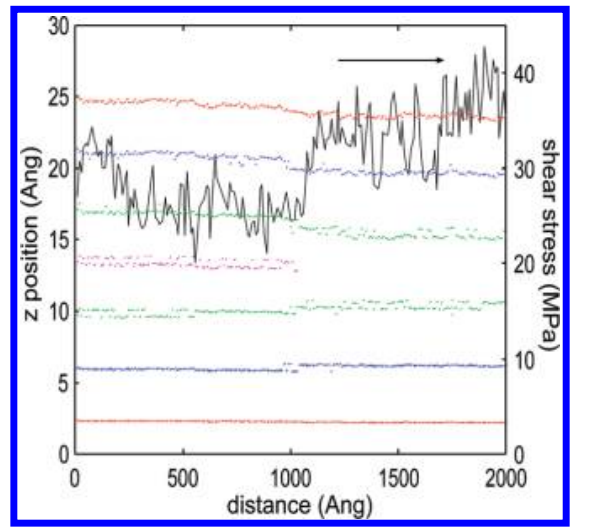

Figure 6. Shear stress and the positions in the $z$-direction of the layers for the $\mathrm{C}_{100} \mathrm{H}_{202}$ system as a function of the sliding distance $d$. The sliding velocity of the block is changed from 100 to $10 \mathrm{~m} / \mathrm{s}$ at $d=0$.

polymer film. For this case we have investigated the influence of the sliding speed on the shear stress. Figure 5 shows the results obtained for a number of linear alkanes with chain length from $20 \mathrm{C}$ atoms to $1400 \mathrm{C}$ atoms. Note that for $\mathrm{C}_{1400} \mathrm{H}_{2802}$ the shear stress is independent of the sliding velocity, whereas the $\mathrm{C}_{20} \mathrm{H}_{42}$ is more liquidlike, with a shear stress approximately linearly related to the sliding velocity.

For the mid-sized molecules (with 60 to $140 \mathrm{C}$ atoms in the chain), for small and large velocities the shear stress is nearly proportional to the logarithm of the sliding velocity, with a small slope at low velocities and a larger one at large velocities. In the range of from 20 to $40 \mathrm{~m} / \mathrm{s}$ the shear stress decreases with increasing sliding velocity. This is due to a layering transition, where the number of layers increases from 6 to 7 . This is proved in Figure 6 which shows the positions in the $z$-direction of the layers for the $\mathrm{C}_{100} \mathrm{H}_{202}$ system as a function of the sliding distance $d$. At $d=0$ the sliding velocity of the block is changed from 100 to $10 \mathrm{~m} / \mathrm{s}$, and after some relaxation time period (which corresponds to the sliding distance $d \approx 1000 \AA$ ) the system abruptly switches from 7 to 6 layers. At the same time the shear stress increases abruptly (see Figure 6). The latter is, at least in part, due to the decreased number of slip planes. The layering transition in Figure 6 is reversible: increasing the velocity back to $100 \mathrm{~m} / \mathrm{s}$ results in a return to seven layers.

As pointed out above, for the mid-sized molecules, for small and large velocities the shear stress is nearly proportional to the logarithm of the sliding velocity, with a small slope at low

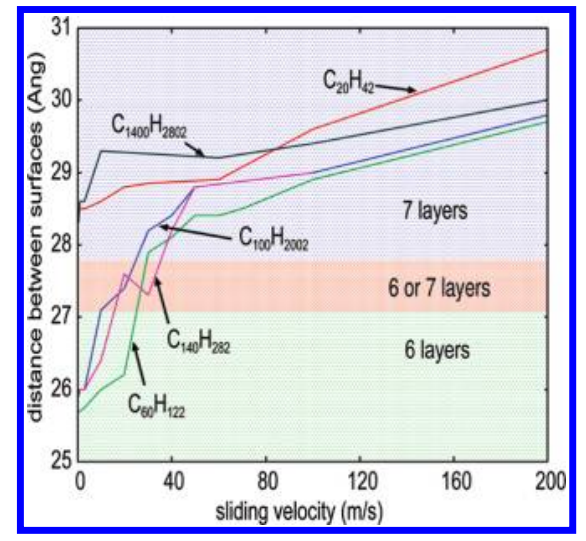

Figure 7. Distance between the surfaces as a function of the sliding velocity for all the investigated systems. There is also an indication of the number of layers present at different surface separations. The normal pressure is $10 \mathrm{MPa}$.

velocities and a larger one at large velocities. The logarithmic velocity dependence is expected for thermally activated stress induced processes, which predict the frictional shear stress ${ }^{6}$

$$
\sigma_{\mathrm{f}} \sim \frac{k_{\mathrm{B}} T}{E_{\mathrm{B}}} \log \left(\frac{v}{v_{0}}\right)
$$

where $k_{\mathrm{B}} T$ is the thermal energy ( $T$ is the temperature), $E_{\mathrm{B}}$ is an energy barrier (activation energy for some rearrangement process involved in lateral slip, e.g., removal of polymer bridge), and $v_{0}$ is a reference velocity. Thus, the larger slope of the $\sigma_{\mathrm{f}}(\log v)$ relation for high slip velocities, as compared to low slip velocities, can be explained by assuming that the energy barrier $E_{\mathrm{B}}$ is smaller in the more open structure, which prevail after the transition from 6 to 7 layers with increasing velocity. We note that the shear stress in Figure 5 has been studied over a rather limited velocity range, and it may also be possible to fit the data with a power relation in the velocity or shear rate. Such power-law behavior has been predicted for the rheology of many soft materials exhibiting structual disorder and metastability. ${ }^{32}$

The change in the number of layers in the film is thermally activated, since no change in the number of layers (on the time scale of our simulations) occurs when the thermostat is at $0 \mathrm{~K}$. Experimental data suggest that the layering transition happens at lower sliding velocities when the normal pressure is decreased. ${ }^{13}$ This is in good accordance with our results as a decrease in pressure from 10 to $3 \mathrm{MPa}$ shifted the transition velocity of the $\mathrm{C}_{60} \mathrm{H}_{122}$ system from about 30 to $20 \mathrm{~m} / \mathrm{s}$.

We will now study the nature of the layering transition in greater detail. In Figure 7 we show the separation between the surfaces for each polymer system we have studied as a function of the sliding speed. Note that the surface separation in the case of $\mathrm{C}_{20} \mathrm{H}_{42}$ and $\mathrm{C}_{1400} \mathrm{H}_{2802}$ increases with increasing sliding velocity with a constant slope, but the number of polymer layers is constant (at seven) in these cases. For the mid-sized molecules there is an abrupt increase in the surface separation in the transition regime of Figure 5. The number of layers is six before the transition (below $10 \mathrm{~m} / \mathrm{s}$ ) and seven after (above $40 \mathrm{~m} / \mathrm{s}$ ).

Let us now study the number of molecular bridges between the layers, which may be affected by the layering transition. ${ }^{12-15}$ There is no exact definition of a molecular bridge, but we have chosen to define a bridging atom by the fact that it does not

(32) Sollich, P.; Lequeux, F.; Hebraud, P.; Cates, M. E. Phvs. Rev. Lett. 1997, 78,2020 . 


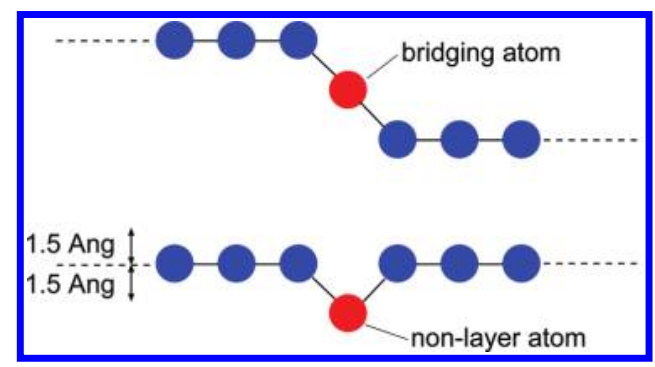

Figure 8. Definitions of bridge and nonlayer atoms used in the present paper.

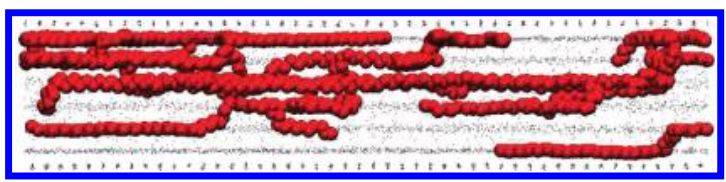

Figure 9. Snapshot picture of a $\mathrm{C}_{100} \mathrm{H}_{202}$ system where 10 randomly chosen molecules are shown. The rest of the atoms are reduced to points. The picture shows that the molecules have atoms in several layers. Some of these segments form bridges, but others are just present in different layers without linking these. The normal pressure is $10 \mathrm{MPa}$, and the sliding velocity is $0.3 \mathrm{~m} / \mathrm{s}$.

belong to the same layer as the preceding atom in the molecule, and at least three of the preceding and three of the following atoms in the molecule are in two different layers (see Figure 8). The bridging phenomenon can be observed in the snapshot shown in Figure 9. The number of bridging atoms as a function of the sliding velocity is shown in Figure 10. Note that the $\mathrm{C}_{1400} \mathrm{H}_{2802}$ system has the same number of bridges through the whole velocity range. The $\mathrm{C}_{20} \mathrm{H}_{42}$ system shows a linear dependence, whereas the mid-sized molecules seem to have a constant number before the transition regime, increasing to a higher level after this regime. The latter can be explained by the more open structure of the seven-layer systems, which makes it easier for polymer molecules from one layer to have segments extending into other layers. Figure 9 also shows that some atoms are outside the center line of the polymer layers without linking these. We define such a nonlayer atom as one having a distance of at least $1.5 \AA$ to the center line of any layer (see Figure 8). We present the number of nonlayer atoms as a function of the sliding velocity in Figure 11.

Figure 11 shows that the $\mathrm{C}_{1400} \mathrm{H}_{2802}$ system has an increasing number of nonlayer atoms with increasing sliding velocity. In the case of $\mathrm{C}_{20} \mathrm{H}_{42}$ the number of nonlayer atoms is proportional to the sliding velocity. The increase in the number of nonlayer atoms is associated with the increase in the separation between the layers with increasing sliding velocity (see Figure 7), which allow polymer segments in a layer to displace away from the layer plane by a considerable distance without experiencing a large repulsion from the nearby polymer layer. One may alternatively interpret the increase in the layer separation as resulting from the increased repulsion from the nonlayer atoms with increasing sliding velocity. In this picture the increased number of nonlayer atoms results from the increased momentum transfer in collisions between atoms in two nearby layers as the sliding velocity increases: these collisions kick polymer segments away from the layer-plane.

The $\mathrm{C}_{60} \mathrm{H}_{122}, \mathrm{C}_{100} \mathrm{H}_{202}$, and the $\mathrm{C}_{140} \mathrm{H}_{282}$ systems exhibit a slow increase in the number of nonlayer atoms before the transition and a faster one when the polymer film has increased to seven layers. Note that the $\mathrm{C}_{100} \mathrm{H}_{202}$ system exhibits a maximum in the number of nonlayer atoms around $10 \mathrm{~m} / \mathrm{s}$. This is caused by a strong fluctuation which sometimes was observed also for the $60 \mathrm{C}$ atom and $140 \mathrm{C}$ atom systems (not shown). This is not

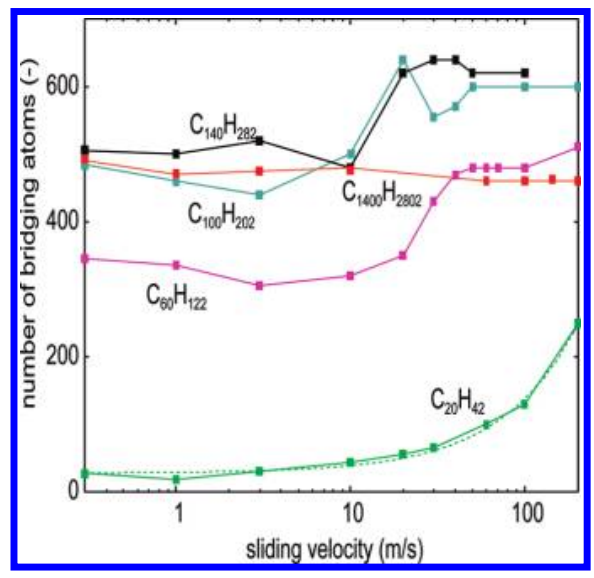

Figure 10. Number of bridging atoms as a function of the sliding velocity for the investigated systems. A bridging atom is defined in the text and in Figure 8. The dotted line is a linear fit to $\mathrm{C}_{20} \mathrm{H}_{42}$. The normal pressure is $10 \mathrm{MPa}$.

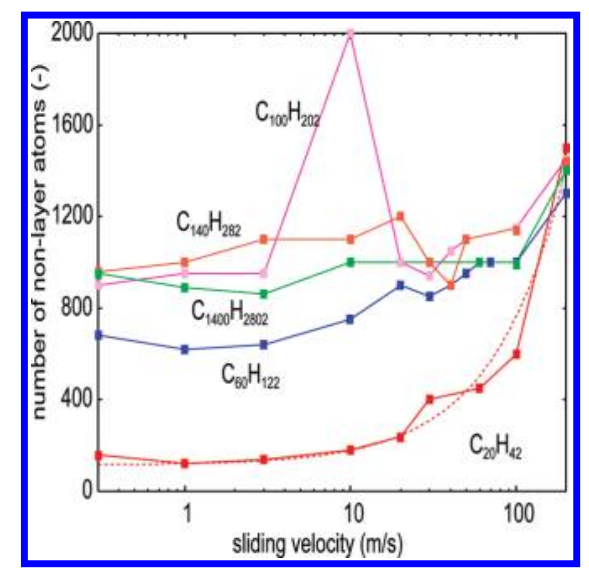

Figure 11. Number of nonlayer atoms as a function of the sliding velocity for the investigated systems. A nonlayer atom is defined in the text and in Figure 8. The dotted line is a linear fit to $\mathrm{C}_{20} \mathrm{H}_{42}$. The normal pressure is $10 \mathrm{MPa}$.

unexpected as strong fluctuations often occur close to phase transition points (in this case a layering transition).

The shift from six to seven layers increases the number of slip planes by one. At the same time the density of bead units in the layers decreases so that the space between the bead units in each layer increases. The increased space reduces the energetic barriers for polymer segment rearrangement processes and results in the formation of more cross-links (see Figure 11). At low velocities the shear stress increases slowly with the increase of the sliding velocity, but after the transition the slope becomes steeper (see Figure 5). This can again be attributed to reduced energetic barriers for polymer rearrangement processes.

Let us now compare the transition observed in Figure 5 with what happens during melting of the polymer film. We have investigated the behavior of the film when it is melted by raising the temperature from 300 to $450 \mathrm{~K}$ (see Figure 12). The figure shows the shear stress and the distance between the surfaces as a function of the sliding time. For both 3 and $10 \mathrm{~m} / \mathrm{s}$ the shear stress decreases to about $10 \mathrm{MPa}$, a much lower level than observed in Figure 5 . At $3 \mathrm{~m} / \mathrm{s}$ the film keeps its six layers as the melting increases the distance between the surfaces to about $27.4 \AA$, a result that is consistent with Figure 7. The combination of a higher sliding velocity and melting increases the distance between the surfaces to about $29 \AA$ when the sliding velocity is $10 \mathrm{~m} / \mathrm{s}$. The film then passes to seven layers as predicted by Figure 7 . 


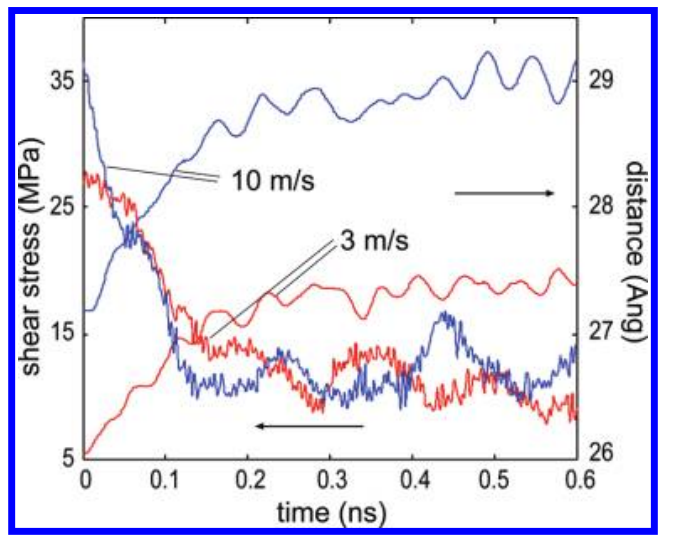

Figure 12. Shear stress and the distance between the surfaces as a function of the sliding time when the temperature is increased from 300 to $450 \mathrm{~K}$ at $t=0$. The melting point is $390 \mathrm{~K}$. For the sliding velocities 3 and $10 \mathrm{~m} / \mathrm{s}$. During melting the film increases its number of layers from 6 to 7 at $10 \mathrm{~m} / \mathrm{s}$, whereas it stays at 6 layers at $3 \mathrm{~m} / \mathrm{s}$. The system is $\mathrm{C}_{100} \mathrm{H}_{202}$ and the normal pressure is $10 \mathrm{MPa}$.

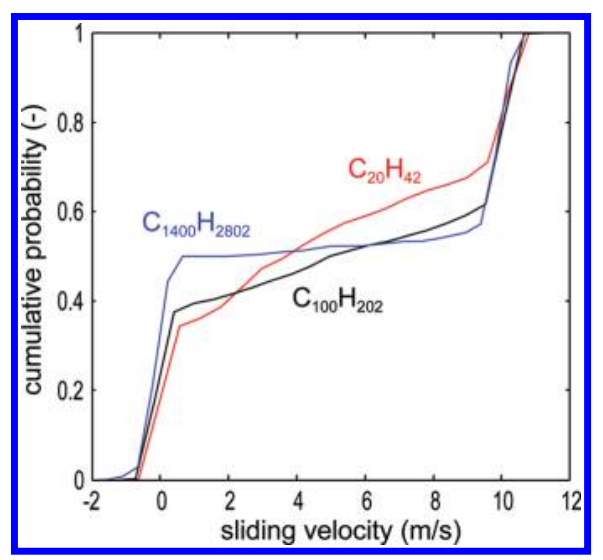

Figure 13. Sliding velocity of atoms as a function of the number of atoms sliding at less than this specific sliding velocity. The number of atoms is accumulated so that the total reach 20000 for $\mathrm{C}_{20} \mathrm{H}_{42}$ and $\mathrm{C}_{100} \mathrm{H}_{202}$, whereas the $\mathrm{C}_{1400} \mathrm{H}_{2802}$ system only has 19600 atoms. The curves are results of an averaging over $0.5 \mathrm{~ns}$. The sliding velocity of the block is $10 \mathrm{~m} / \mathrm{s}$, and the normal pressure is $10 \mathrm{MPa}$.

As discussed above, for the $\mathrm{C}_{20} \mathrm{H}_{42}$ system at room temperature we observe liquidlike behavior except at very low sliding velocities (see Figure 5). Similarly for the $\mathrm{C}_{100} \mathrm{H}_{202}$ system, as the temperature increases above the melting temperature the friction drops abruptly (Figure 12). If one deduces effective viscosities using the definition $\eta_{\text {eff }}=\sigma_{\mathrm{f}} d / v$, one obtains for the $20 \mathrm{C}$ system (Figure 5) $\eta_{\mathrm{eff}} \approx 0.001 \mathrm{~Pa}$ s and for the $100 \mathrm{C}$ system (Figure 12) at $v=3 \mathrm{~m} / \mathrm{s}$ $\eta_{\mathrm{eff}} \approx 0.01 \mathrm{~Pa}$. The viscosities of linear hydrocarbon fluids (at the melting point) increase approximately linearly ${ }^{33}$ with the number of chain $\mathrm{C}$ atoms up to several hundred $\mathrm{C}$ atoms (and after that faster due to chain entanglement). For $\mathrm{C}_{10} \mathrm{H}_{22}$ at the melting temperature $\eta \approx 0.001 \mathrm{~Pa}$ s. Thus, the values we obtain in the simulations for confined films agree within a factor of $\sim 2$ with the bulk viscosity values. We note that some SFA experiments on highly confined lubricant films have detected strongly enhanced effective viscosities and strong shear thinning with increasing strain rate. These experiments involve much lower shear velocities than can be obtained in MD simulations. In our simulations we have also some indication of shear thinning: in Figure 5 the shear

(33) Tabor, D. Philos. Mag. 1988, A57, 217.

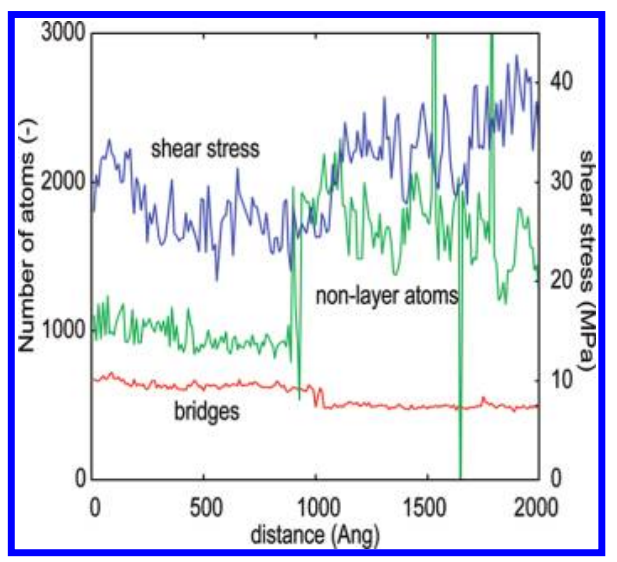

Figure 14. Number of bridging and nonlayer atoms and the shear stress as a function of the sliding distance for $\mathrm{C}_{100} \mathrm{H}_{202}$. The sliding velocity of the block is changed from 100 to $10 \mathrm{~m} / \mathrm{s}$ at $0 \AA$.

stress for the $\mathrm{C}_{20} \mathrm{H}_{42}$ system does not decrease toward zero for decreasing sliding velocity but levels off at a constant value, indicating solidlike behavior at very low sliding velocities. Similarly in Figure 12 the shear stress is rather similar for the velocities 3 and $10 \mathrm{~m} / \mathrm{s}$. See also ref 34 for a discussion of the effective viscosity of confined hydrocarbon films.

Let us study the velocity profiles of the systems. Figure 13 shows the cumulative velocity probability distribution for systems sliding at $10 \mathrm{~m} / \mathrm{s}$. Note that for the $\mathrm{C}_{1400} \mathrm{H}_{2802}$ system nearly all the slip occurs at one interface at the center of the polymer film. Thus, this system can be considered as two polymer slabs sliding against each other. This picture also explains the independence of the shear stress for $\mathrm{C}_{1400} \mathrm{H}_{2802}$ to the number of bridges (Figure 10) and nonlayer atoms (Figure 11), since these are presumably mostly appearing inside the polymer slabs and thus do not influence the interfacial slip. The $\mathrm{C}_{20} \mathrm{H}_{22}$ molecules have their sliding planes distributed over the whole thickness of the film. This is expected for a liquidlike flow, but Figures 10 and 11 indicate that this also could be a result of an increasing number of bridges and/or nonlayer atoms. The mid-sized molecules in Figure 13 have a velocity profile in between the longest molecules and the shortest ones and can be considered as a so-called plug flow, where the outermost layers are pinned to the surfaces of the block and the substrate.

In Figure 14, at $d=0 \AA$ (where $d$ is the slip distance) the sliding velocity of the block is changed from 100 to $10 \mathrm{~m} / \mathrm{s}$. Note that as the film relaxes the number of bridges and nonlayer atoms decreases slightly, and so does the shear stress. When the transition regime is reached, the number of nonlayer atoms suddenly increases as the central layers become mobile and (in the $z$-direction) diffuse. The increase in shear stress follows this increase in the number of nonlayer atoms whereas the number of bridges decreases to a lower constant level as the number of layers goes from 7 to 6 . This can be understood as the density of molecules in the layers is smaller for the 7-layer state than for the 6-layer state, and hence the ability for chain molecules to (due to a fluctuation) interdiffuse and form bridges will be largest in the 7-layer state.

\section{Summary and Conclusions}

We have presented results of molecular dynamics calculations of friction for two solids separated by $\mathrm{a} \approx 3 \mathrm{~nm}$ thick polymer film. Two types of systems were considered: (a) a polymer film pinned

(34) Manias, E.; Bitsanis, I.; Hadziioannou, G.; Brinke, G. T. Europhys. Lett. 1996, 33, 371. 
to one of the solid surfaces and sliding at the other solid surface (the "metal"-polymer case): (b) the polymer layers pinned to both solid surfaces and shearing at the polymer-polymer interface (the polymer-polymer case). We used linear alkane molecules with the number of carbon atoms 20,60, 100, 140, and 1400.

The frictional shear stress for the polymer-polymer systems is much higher than for the "metal"-polymer systems. This is due to the same size of the atoms or molecules on both sides of the slip plane for the polymer-polymer case, resulting in strong interlocking (as for a commensurate interface), while the "metal"polymer interfaces are incommensurate. (The lattice constant of the "metal" substrate is different from the distance between atoms of the lubricant molecules.)

We have studied the velocity dependence of the frictional shear stress for both cases. In the first setup the shear stresses are relatively independent of molecular length. For the shortest hydrocarbon $\mathrm{C}_{20} \mathrm{H}_{42}$ the frictional shear stress is lower and increases approximately linearly with the velocity.

For polymer sliding on polymer the friction is significantly larger, and the velocity dependence is more complex. For the longest molecules (1400 carbon atoms) the shear stress is independent of the sliding velocity as the sliding occurs primarily at one interfacial slip plane. The shortest molecules again exhibit liquidlike sliding with the shear stress being approximately proportional to the sliding velocity. The mid-sized molecules (60-140 C atoms) show a slightly increasing shear stress at low velocities and a faster increase at high sliding velocities. Between these regimes there is a transition with a decrease in the shear stress with increasing sliding velocity.

The mechanism behind this behavior seams to be a kinetic phase transition involving an abrupt increase in the number of layers in the film, with increasing sliding velocity, which decreases the shear stress abruptly. Further increase of the sliding velocity will increase the shear stress rapidly, which we attribute to the interaction between the layers via nonlayer atoms.

Acknowledgment. A part of the present work was carried out in frames of the European Science Foundation EUROCORES Programme FANAS supported from the EC Sixth Framework Programme, under Contract N. ERAS-CT-2003-980409. I.M.S. and V.N.S. acknowledge support from IFF, FZ-Jülich, as well as hospitality and help of the staff during their research visits. 